Check for updates

Cite this: RSC Adv., 2019, 9, 917

Received 15th November 2018 Accepted 26th December 2018

DOI: $10.1039 / c 8 r a 09410 a$

rsc.li/rsc-advances

\title{
Characterization of biochars derived from different materials and their effects on microbial dechlorination of pentachlorophenol in a consortium $\dagger$
}

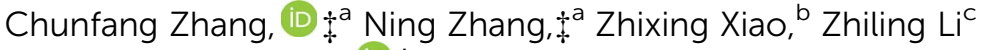 \\ and Dongdong Zhang (D) *a
}

\begin{abstract}
The properties of biochars derived from different raw materials (rice husk, bamboo, caragana, and garbage) and their effects on the microbial reductive dechlorination of pentachlorophenol (PCP) were investigated to understand how biochars influence the biotransformation of environmental pollutants. The results indicated that only caragana-derived biochar showed stable electron transfer activity for PCP dechlorination. Electro(chemical) analyses revealed that caragana biochar had the highest electrical conductivity (EC) $\left(2.22 \times 10^{6} \mu \mathrm{S} \mathrm{cm}^{-1}\right)$, while those of the other biochars were $<1500 \mu \mathrm{Sm}^{-1}$. The electron transfer capacities were within the ranges of $61.63-155.83 \mu \mathrm{mol} \mathrm{e}^{-} \mathrm{g}^{-1}$. Cyclic voltammetry analysis suggested that there were no obvious redox peaks for the biochars, while the Fourier transform infrared analysis showed similar transmission spectra with variable absorption intensity; this suggested that all biochars possessed similar structures and functional group classes and the enhancement of PCP dechlorination was not attributable to the redox reaction. Overall, the beneficial effects of caragana biochar on PCP dechlorination depended on the EC rather than the redox functional groups, possibly because high EC values enabled the highest electron transfer, and thus resulted in the greatest promotion of reductive dechlorination activity.
\end{abstract}

\section{Introduction}

Pentachlorophenol (PCP) is a typical halogenated aromatic compound that has been used widely as an insecticide, wood preservative, and anti-mold agent. It has been banned due to the threat that it poses to human health, including its high toxicity to the liver and kidneys and its probable carcinogenicity, as well as the ecological environment. ${ }^{1}$ Anaerobic bioremediation has attracted attention as a potential cost-effective technology for detoxifying PCP. $^{2-4}$ However, the microbial anaerobic dechlorination of halogenated aromatic compounds including PCP typically occurs in the presence of soils/sediments ${ }^{5}$ or the solid-phase humins extracted from soils/sediments, ${ }^{2,6,7}$ which function as a solid-phase electron shuttle for the promotion of reductive dehalogenation.

\footnotetext{
${ }^{a}$ Institute of Marine Biology, Ocean College, Zhejiang University, Zhoushan 316021, Zhejiang, China. E-mail: zhangdongzju@zju.edu.cn; Tel: +8613522550565

${ }^{b}$ College of Urban Construction, Nanjing Tech University, Nanjing 211800, China

${ }^{c}$ State Key Laboratory of Urban Water Resources and Environment, School of Environment, Harbin Institute of Technology, Harbin 150090, China

$\dagger$ Electronic supplementary information (ESI) available. See DOI: 10.1039/c8ra09410a

\$ These authors contributed equally to this study and are joint first authors.
}

Biochar is a refractory, stable, highly aromatic, carbon-rich solid material produced by the slow pyrolysis of diverse biomass species under low-oxygen conditions. It shows great potential in carbon sequestration, and can mediate greenhouse gas emissions, improve soil fertility, and remediate environmental pollution. ${ }^{8-10}$ Due to its porous, large internal surface area and high aromaticity, biochar has also been reported to enhance the adsorption of various organic and metal pollutants and effectively immobilize these environmental pollutants, thereby reducing their bioavailability and ecotoxicity. ${ }^{11-15}$ Many studies have investigated the application of biochar or modified biochar for the removal of organic and metal pollutants; however, most of these studies have focused on its adsorption capacity. For example, biochar obtained from cottonseed hulls exhibits a very high adsorption affinity for various pesticides, ${ }^{\mathbf{1 3}}$ cow manure biochar was utilized as a potential adsorbent to remove oxytetracycline and carbaryl from aqueous solutions, ${ }^{\mathbf{1 2}}$ and dairy-manure biochar can immobilize lead and atrazine in soil to reduce their toxicity. ${ }^{\mathbf{1 1}}$ In addition, modified biochar with nanoscale zero-valent iron and silver showed higher carbon tetrachloride removal efficiency than the original biochar as a novel adsorbent. ${ }^{\mathbf{1 4}}$

Recently, biochar has been reported as a solid-phase electron shuttle to support microbial redox transformations. ${ }^{16-19}$ Studies 
have shown that plant biomass-derived biochar had high capacities to accept and donate electrons, ${ }^{\mathbf{1 8}}$ rice straw-derived biochar significantly accelerated electron transfer from Geobacter sulfurreducens cells to halogenated compounds for enhancing dechlorination, ${ }^{\mathbf{1 9}}$ and poultry litter and wastewater biosolids-derived biochar could shuttle electrons to promote the reductive removal of nitro herbicides and explosives, ${ }^{17}$ which were potentially due to graphene moieties, surface functional groups, or redox-active metals harbored in biochar. ${ }^{17}$ Moreover, biochar derived from pine could promote direct interspecies electron transfer in co-cultures of Geobacter metallireducens with Geobacter sulfurreducens or Methanosarcina barkeri via a conduction-based mechanism, in which electrons migrate through the biochar from electron-donating to electron-accepting cells. ${ }^{\mathbf{1 6}}$ As solid-phase electron shuttles, humins that originated from different sources exhibited different redox activity for microbial reactions, especially for halogenated aromatic compound dechlorination; some humins even exhibited no dechlorination activity. ${ }^{\mathbf{2 0 2 1}}$ Similarly, biochars derived from different raw materials also exhibit very different physicochemical properties. Because of these characteristics, biochars derived from different materials might exhibit different influences on microbial transformations. Nevertheless, how raw materials influence the effects of biochars on microbial redox transformation has not yet been thoroughly investigated.

In this study, we characterized biochars produced from four different raw materials-rice husk, bamboo, caragana, and garbage-via chemical, electro(chemical), and spectroscopic methods to gain insight into their properties and differences in terms of PCP microbial transformation. This study is helpful for us to understand the electron transfer process of microbial redox reactions mediated by biochar and the impact of the raw material used for biochar on the bioremediation of environmental contaminants.

\section{Materials and methods}

\subsection{Biochar preparation and elemental characterization}

In order to produce biochar, rice husk, bamboo, caragana, and garbage (household waste) were first cut into 1-2 cm pieces, and then placed into a cylindrical quartz tube in an electric furnace and pyrolyzed at $500{ }^{\circ} \mathrm{C}$ for $4 \mathrm{~h}$ under an $\mathrm{N}_{2}$ flow of $1.5 \mathrm{~L} \mathrm{~min}^{-1}$. After cooling to room temperature, the charred materials were milled to approximately $0.15 \mathrm{~mm}$ and sieved through a 100mesh sifter. ${ }^{19}$ The $\mathrm{C}, \mathrm{H}, \mathrm{N}$, and ash contents of the biochars were determined using a Yanaco MT-5 CHN-corder (Yanaco New Science Inc., Japan). Finally, the $\mathrm{O}$ contents were calculated based on the mass difference.

\subsection{Influence of biochar on microbial reductive dechlorination of PCP}

An anaerobic humin-dependent PCP-to-phenol dechlorinating mixed culture was used as the inoculum source in this study, ${ }^{2}$ which had been kept in our laboratory for years. This PCPdechlorinating mixed culture contains Sulfurospirillum,
Clostridium, and Dehalobacter, which were thought to be involved in the reductive dechlorination of $\mathrm{PCP}^{2,4}$ The biochar culture in this study was obtained by transferring $5 \%(\mathrm{v} / \mathrm{v})$ of the inoculum source to fresh medium using biochar as a substitute for humin, and was maintained at $30{ }^{\circ} \mathrm{C}$ through subculture at an interval of 2-3 weeks.

For the preparation of the biochar cultures, $50 \mathrm{~mL}$ serum bottles containing $30 \mathrm{~mL}$ of mineral medium, $1 \%(\mathrm{w} / \mathrm{v})$ biochar, $15 \mathrm{mM}$ formate, and $20 \mu \mathrm{M}$ PCP were flushed with $\mathrm{N}_{2}$. The bottles were sealed using Teflon-coated butyl rubber stoppers and aluminum seals, and autoclaved at $121{ }^{\circ} \mathrm{C}$ for $20 \mathrm{~min}$. The mineral medium consisted of (per liter) $1.0 \mathrm{~g}$ of $\mathrm{NH}_{4} \mathrm{Cl}, 0.05 \mathrm{~g}$ of $\mathrm{CaCl}_{2} \cdot 2 \mathrm{H}_{2} \mathrm{O}, 0.1 \mathrm{~g}$ of $\mathrm{MgCl}_{2} \cdot 6 \mathrm{H}_{2} \mathrm{O}, 0.4 \mathrm{~g}$ of $\mathrm{K}_{2} \mathrm{HPO}_{4}, 1 \mathrm{~mL}$ of trace element SL-10 solution, ${ }^{22} 1 \mathrm{~mL}$ of Se/W solution, ${ }^{22} 15 \mathrm{mM}$ MOPS buffer ( $\mathrm{pH} 7.2$ ), and $50 \mathrm{mg} \mathrm{L}^{-1}$ resazurin-Na. The influence of the biochars from different materials on the microbial PCP dechlorination activity was examined by an incubation experiment conducted in the same way as the culture maintenance. The different biochars were added at the same concentration. After appropriate incubation periods (15 days), PCP and its metabolites in each culture were analyzed using a QP5050 gas chromatograph-mass spectrometer (Shimadzu, Japan) equipped with a DB-5MS column (J\&W Scientific, USA). The influence of biochar on PCP dechlorination was evaluated after at least two culture transfers into new medium. Additionally, a biological control without biochar but with inoculum and an abiotic control without inoculum but with each type of biochar were examined. All experiments were performed in triplicate.

\subsection{Adsorption of PCP by biochars}

PCP was added at a concentration of $20 \mu \mathrm{M}$ in $20 \mathrm{~mL}$ serum bottles containing $15 \mathrm{~mL}$ of mineral medium with or without biochars $\left(10 \mathrm{~g} \mathrm{~L}^{-1}\right)$ under the same conditions as mentioned above for culture maintenance. The medium was sampled after appropriate intervals (i.e., 1, 3, 10, 18, 24, 48, 72, and 96 hours), then the biochar was removed by centrifugation at $12000 \mathrm{rpm}$ for $6 \mathrm{~min}$. The concentration of PCP in the supernatant was extracted and analyzed as mentioned above.

\subsection{Spectroscopic characterization of biochar}

Fourier transform infrared (FTIR) spectra of the biochars were determined using a spectrometer (6100, JASCO, Japan) in the 400-4000 $\mathrm{cm}^{-1}$ range at a resolution of $4 \mathrm{~cm}^{-1}$ and eight accumulation modes. The $\mathrm{KBr}$ pellet technique was used as described previously. ${ }^{20}$

\subsection{Analysis of the electrical conductivity of biochar}

The electrical conductivity (EC) of biochar was measured using the two-probe bed technique consisting of a pressure device, a hand-held milliohm meter, and a cylindrical iron bed. The inner surface of the bed was completely insulated from the biochar and pistons by an embedded polypropylene material. After placing roughly $1.0 \mathrm{~g}$ of biochar sample into the bed, high pressure $(4 \mathrm{MPa})$ was applied, and the resistance of the whole bed was recorded. ${ }^{19}$ 


\subsection{Electrochemical analyses of biochar}

The electrochemical experiments were performed using a potentiostat (HSV-110; Hokuto Denko Inc., Japan), comprised of a biochar-adhering graphite rod (5 $\mathrm{mm}$ in diameter), a $\mathrm{Pt}$ wire, and a $\mathrm{Ag} / \mathrm{AgCl}$ electrode as the working, counter, and reference electrodes, respectively. For the working electrode, $0.03 \mathrm{~g}$ of biochar was uniformly adhered on the surface of graphite rod (effective surface: $6.48 \mathrm{~cm}^{2}$ ) using carbonconductive tape (SPI Supplies, USA). Switchable potentials $(-0.7 \mathrm{~V}$ and $+0.5 \mathrm{~V}$ ( $v s$. $\mathrm{Ag} / \mathrm{AgCl}))$ were applied to the working electrode to determine the electron accepting capacity (EAC) and electron donating capacity (EDC) of the adhered biochar in mineral medium (as the electrolyte) under constant stirring and $\mathrm{N}_{2}$ flow conditions as described by Yuan et al. with minor modifications. ${ }^{23}$ The total electron transfer capacities (ETCs) of the biochars, representing the total capacity to donate and accept electrons, were obtained from the sum of the EACs and EDCs. ${ }^{18,19}$

For the cyclic voltammetry (CV) analysis, mineral medium also was used as the electrolyte, and CV curves were recorded with a scan rate of $10 \mathrm{mV} \mathrm{s}^{-1}$ and a potential range of -0.6 to $+0.8 \mathrm{~V}$ ( $v s$. Ag/AgCl). All test solutions were purged with highpurity $\mathrm{N}_{2}$ for $15 \mathrm{~min}$ before each set of scans to exclude oxygen interference, and background scans were carried out prior to each measurement.

\section{Results and discussion}

\subsection{Influence of biochar on the microbial dechlorination of PCP}

Fig. 1 and 2 shows the influence of biochar types (carnage, rice husk, bamboo, and garbage) on PCP dechlorination in the enrichment culture; among the four examined biochars, only caragana biochar stably enhanced the microbial dechlorination of PCP. Specifically, PCP was completely dechlorinated into phenol in the culture amended with caragana biochar after incubation for 15 days; the activity continued to be stable after three generations, suggesting that caragana biochar is an effective electron shuttle for microbial PCP-to-phenol dechlorination (Fig. 1a). In contrast, PCP dechlorination activity was not observed in the presence of the other three biochars (Fig. 2), neither in the absence of inoculum or caragana biochar (Fig. 1b). The different biochars showed the comparable adsorption abilities to PCP, 5-7 $\mu \mathrm{M}$ of equilibrium concentrations of PCP after 24 hours, and no dechlorination metabolites were observed (Fig. S5 $\dagger$ ). This suggests that the dechlorination enhancement was due to the microbial metabolism mediated by biochar, that not all types of biochar could enhance the microbial dechlorination of PCP, and both microbial activity and caragana biochar were required for PCP dechlorination. Overall, our results indicate that the activity of biochars for the enhancement of PCP dechlorination varies depending on the raw materials used in their production.

Several studies have demonstrated the importance of solidphase electron shuttle. For example, humic substances immobilized on solid materials (i.e., alumina particles and anion
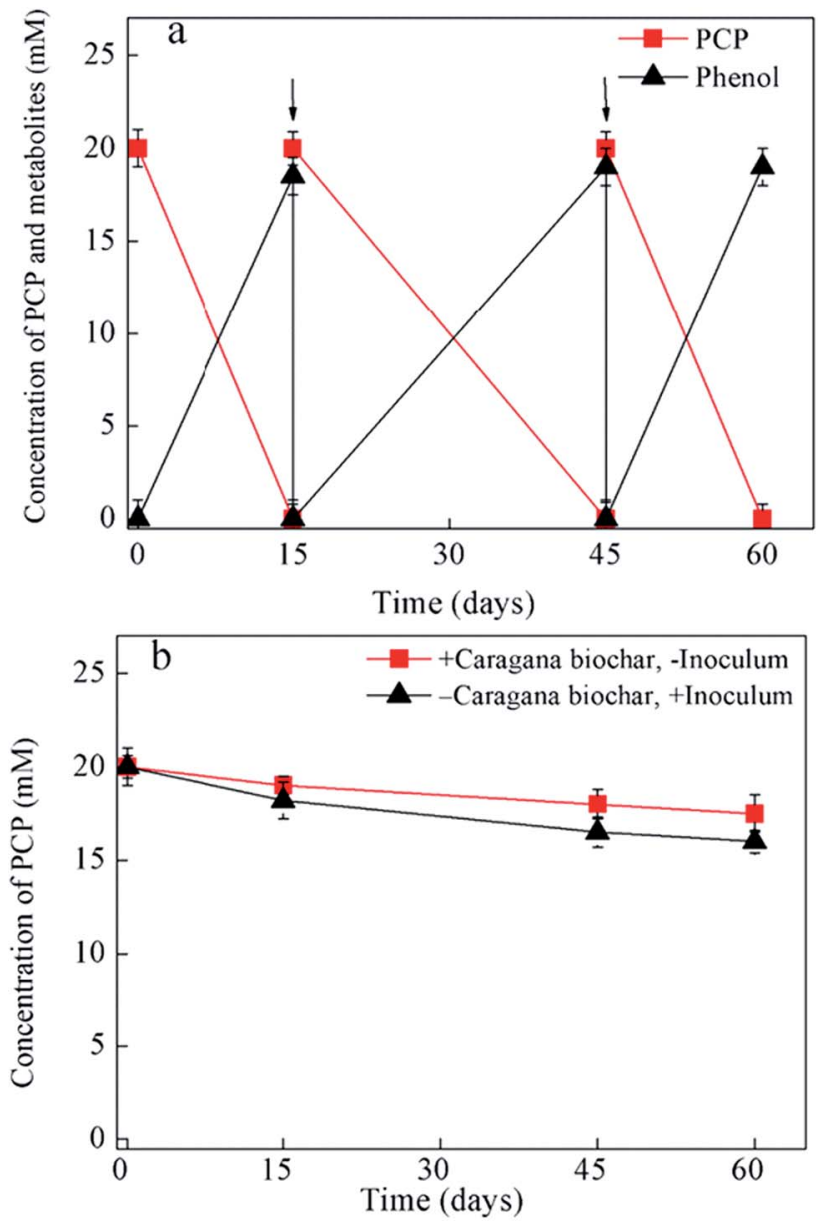

Fig. 1 Influence of caragana biochar on PCP dechlorination based on PCP and final metabolite (i.e., phenol) concentrations in the enrichment culture with (a) both biochar and inoculum and (b) the biological and abiotic controls. The error bars represent the standard deviation of three measurements $(n=3)$.

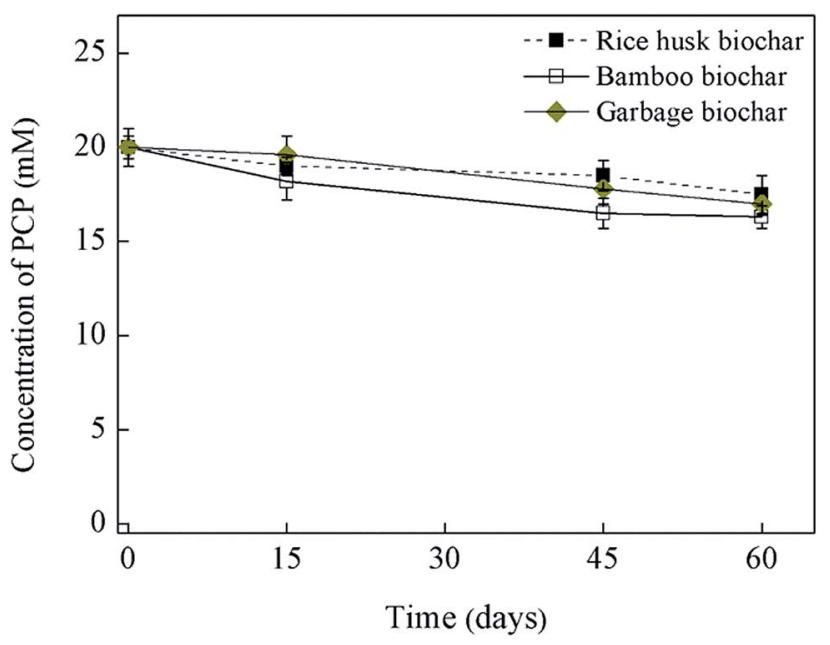

Fig. 2 Influence of other three biochar types (rice husk, bamboo, garbage) on PCP dechlorination concentrations in the enrichment culture. The error bars represent the standard deviation of three measurements $(n=3)$ 
Table 1 Elemental analysis of different biochars

Elemental composition (\%)

\begin{tabular}{lllllrrrr} 
Biochar type & $\mathrm{C}$ & $\mathrm{H}$ & $\mathrm{N}$ & $\mathrm{O}$ & $\mathrm{C} / \mathrm{N}$ & $\mathrm{H} / \mathrm{C}$ & $\mathrm{O} / \mathrm{C}$ & Ash (\%) \\
\hline Rice husk biochar & 51.40 & 1.50 & 0.99 & 14.01 & 60.57 & 0.35 & 0.20 \\
Bamboo biochar & 75.45 & 2.14 & 0.50 & 19.91 & 176.05 & 0.34 & 0.20 \\
Caragana biochar & 47.53 & 1.49 & 0.67 & 25.21 & 82.76 & 0.38 & 0.40 \\
Garbage biochar & 30.74 & 0.94 & 0.62 & 16.51 & 57.84 & 0.37 & 0.40
\end{tabular}

exchange resins) can effectively mediate the reductive biodegradation of organic pollutants. ${ }^{24,25}$ Moreover, humins extracted from different soil and sediment exhibited the ability to stably sustain both PCP dechlorination and tetrabromobisphenol A (TBBPA) debromination in two anaerobic cultures, respectively, whereas HA extracted from the same source in soluble forms was unstable in mediating the activity and the dehalogenation activity was lost after subculture. ${ }^{2,6}$ Additionally, we previously reported that an insoluble Fe-humic acid complex could function as a solid-phase electron shuttle for the enhancement of the microbial reductive dechlorination of PCP. ${ }^{26}$ In the current study, four types of biochars were examined as substitutes for humins in the incubation of this humin-dependent PCP-tophenol culture. Only caragana biochar displayed stable PCPto-phenol dechlorination activity, while the other three biochars exhibited a negative effect, indicating that only caragana biochar could successfully replace humin to maintain the dechlorination activity. Therefore, caragana biochar may contain redox functional groups or other related electrochemical characteristics, which are responsible for this activity. To better understand the effects of biochar types from different sources upon PCP dechlorination activity, the spectroscopic and electrochemical characteristics of biochars were further analyzed.

\subsection{Elemental composition and spectroscopic property of biochars}

The elemental compositions and atomic ratios of the four biochars are shown in Table 1 . The $\mathrm{C}, \mathrm{H}, \mathrm{N}, \mathrm{O}$, and ash contents were in the range of $30.74-75.45 \%, 0.94-2.14 \%, 0.50-0.99 \%$, $14.01-25.21 \%$, and $2.02-51.26 \%$, respectively. The $\mathrm{C} / \mathrm{N}, \mathrm{H} / \mathrm{C}$, and $\mathrm{O} / \mathrm{C}$ atomic ratios were calculated as $57.84-176.05,0.34-$ 0.38 , and $0.20-0.40$, respectively. The low $\mathrm{H} / \mathrm{C}$ ratios $(<1)$ in this study may imply that the biochars had a predominant aromatic nature and a low degree of aliphatic condensation. The $\mathrm{O} / \mathrm{C}$ ratio is often considered as an indicator of O-containing functional groups (e.g., carboxylic acid), where high $\mathrm{O} / \mathrm{C}$ ratios are indicative of high carboxyl-C and carbonyl-C compositions. ${ }^{27}$ Thus, the biochars from caragana and garbage, which both had the highest $\mathrm{O} / \mathrm{C}$ ratios, may have had the most O-containing functional groups.

As shown in Fig. 3, the FTIR spectra of all biochars were similar, although their absorbance intensities varied; the interpretation of these spectra was based on previously published studies. ${ }^{28-30}$ All biochars showed bands from $3415 \mathrm{~cm}^{-1}$ to $882 \mathrm{~cm}^{-1}$ in Fig. 3, which attributed to the $\mathrm{O}-\mathrm{H}$ vibrations of carboxylic or phenolic groups $\left(3415 \mathrm{~cm}^{-1}\right)$, aliphatic $\mathrm{CH}_{3}$ and $\mathrm{CH}_{2}$ groups $\left(2925 \mathrm{~cm}^{-1}\right), \mathrm{N}-\mathrm{H}$ stretching vibration $\left(2516 \mathrm{~cm}^{-1}\right)$, carbonyl vibrations of esters $\left(1797 \mathrm{~cm}^{-1}\right), \mathrm{N}-\mathrm{H}$ groups in amides $\left(1610 \mathrm{~cm}^{-1}\right)$, aliphatic $\mathrm{C}-\mathrm{H}$ groups and $\mathrm{C}=\mathrm{C}$ stretching $\left(1444 \mathrm{~cm}^{-1}\right)$, and aromatic $\mathrm{C}-\mathrm{H}$ bending $\left(882 \mathrm{~cm}^{-1}\right)$, respectively. The FTIR spectra of the biochars were similar, with different absorbance intensities, indicating that they possessed similar structures and contained the same classes of functional groups regardless of the raw materials used for their production, although only caragana biochar could maintain PCP-tophenol activity.

\subsection{Electro(chemical) properties of biochars}

The ETCs of the biochars were evaluated by integrating the current enhancement of the amperometric response at a graphite working electrode. ${ }^{21}$ Negative potentials of $-0.7 \mathrm{~V}$ to the working electrode (positive potentials of $+0.5 \mathrm{~V}$ to EDC) were used to evaluate the EACs (Table S1 $\dagger$ ). In response to negative and positive potentials to the working electrode, current consumption showed immediate increases (Fig. S2 $\dagger$ ), suggesting that redox-active moieties in biochars could be directly reduced and oxidized by the graphite electrode under $-0.7 \mathrm{~V}$ and $+0.5 \mathrm{~V}$. The EACs and EDCs were within the ranges of 22.18$73.98 \mu \mathrm{mol} \mathrm{e} \mathrm{e}^{-} \mathrm{g}^{-1}$ and $27.65-86.49 \mu \mathrm{mol} \mathrm{e} \mathrm{e}^{-} \mathrm{g}^{-1}$, respectively (Table S1†). All biochars had comparable EACs, which were lower than the corresponding EDCs. The low EACs of the

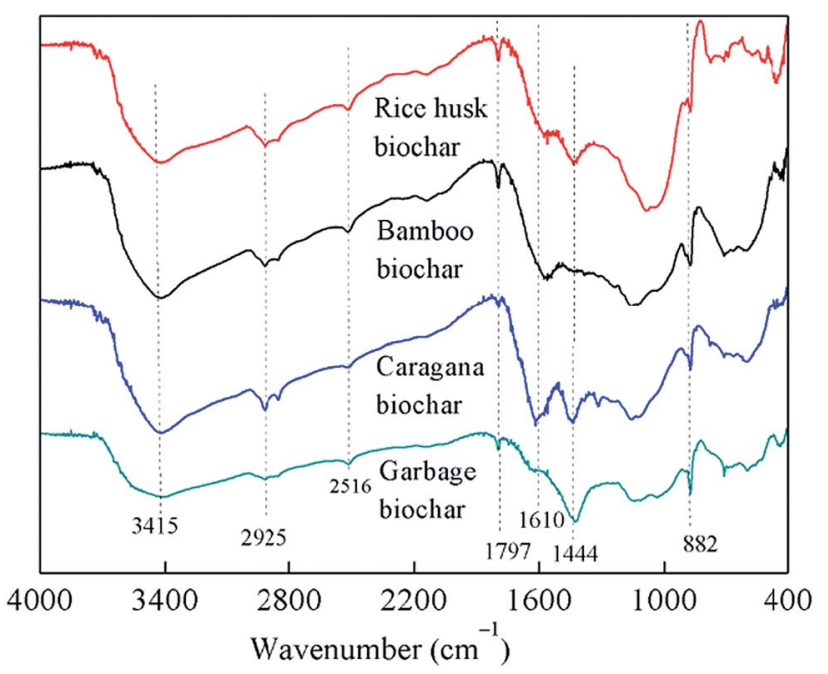

Fig. 3 Fourier transform infrared (FTIR) spectra of the four biochars. 


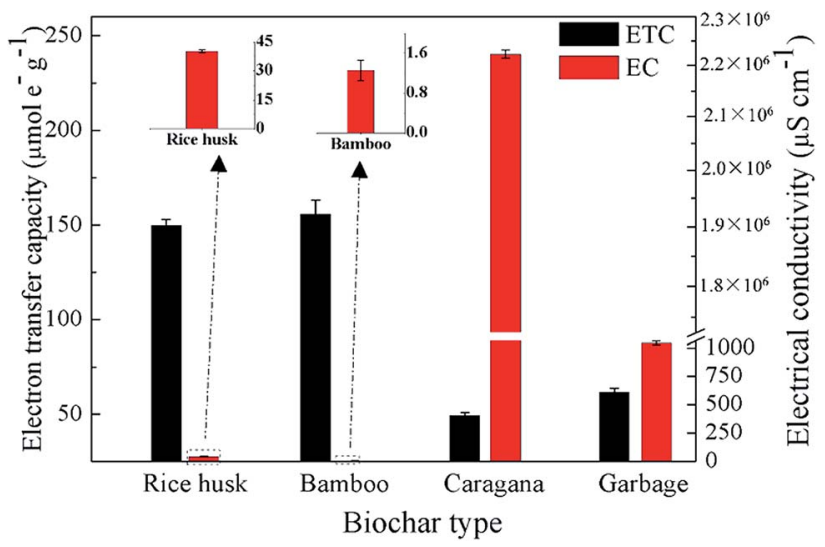

Fig. 4 Changes in the electron transfer capacities (ETCS) and electrical conductivities (ECs) of biochars from different materials. The error bars represent the standard deviation of three measurements $(n=3)$.

biochars indicated that most of the redox-active moieties of the tested biochars were in the reduced forms, which might be due to the production of these biochars under a nitrogen atmosphere condition.

Fig. 4 shows the electron transfer capacities (ETCs) and electrical conductivities (ECs) of the four biochars. The ETCs of the four types of biochars were within the ranges of 49.37$155.83 \mu \mathrm{mol} \mathrm{e}^{-} \mathrm{g}^{-1}$. Caragana biochar, with positive redox activity for microbial PCP dechlorination, exhibited the lowest ETC. Combined with the FTIR analysis of all biochars, the PCP dechlorination activity maintained by caragana biochar was not primarily attributed to the strength of its ETC. The ECs differed greatly among the four biochars, ranging from 1.25 to $2.22 \times$ $10^{6} \mu \mathrm{s} \mathrm{cm}^{-1}$. The EC of caragana biochar, which could maintain the microbial PCP dechlorination, had the highest value $(2.22 \times$ $\left.10^{6} \mu \mathrm{s} \mathrm{cm}^{-1}\right)$. By contrast, the EC values of the other three biochars with no redox activity toward PCP dechlorination were in the range of 1.25 to $1.04 \times 10^{3} \mu \mathrm{s} \mathrm{cm}^{-1}$, which were at least three orders of magnitude lower than that of caragana biochar. Therefore, the EC of biochar, rather than the redox-active moieties, was more important in regard to the maintenance of microbial PCP dechlorination. The differences of ECs among these four types of biochars might due to the different proportions of polycondensed aromatic structures (or conductive graphite regions). Further research in this regard is warranted.

This result was in high agreement with previous studies, which reported that the catalytic activity for the transformation of nitrated explosives by biochar produced from red oak wood ${ }^{31}$ and the promotion of direct interspecies electron transfer by pine-derived biochar ${ }^{16}$ were due to their EC properties rather than their surface functional groups. Although Yu et al. ${ }^{19}$ have reported that both of the surface redox-active moieties and ECs of biochar contribute to the microbial dechlorination of halogenated compounds, the conductive graphite regions of biochars play crucial roles in the electron transfer process of the microbial dechlorination. That is to say, the conductive properties of biochars determined their electron transfer characteristic, and thereby the PCP reductive dechlorination activity. ${ }^{\mathbf{1 9}}$ Additionally, the role of the biochar EC in this study may be similar to that of conductive magnetite nanoparticles ${ }^{32}$ and granular active carbon ${ }^{33}$ reported in previous studies, which demonstrated that magnetite nanoparticles served as an
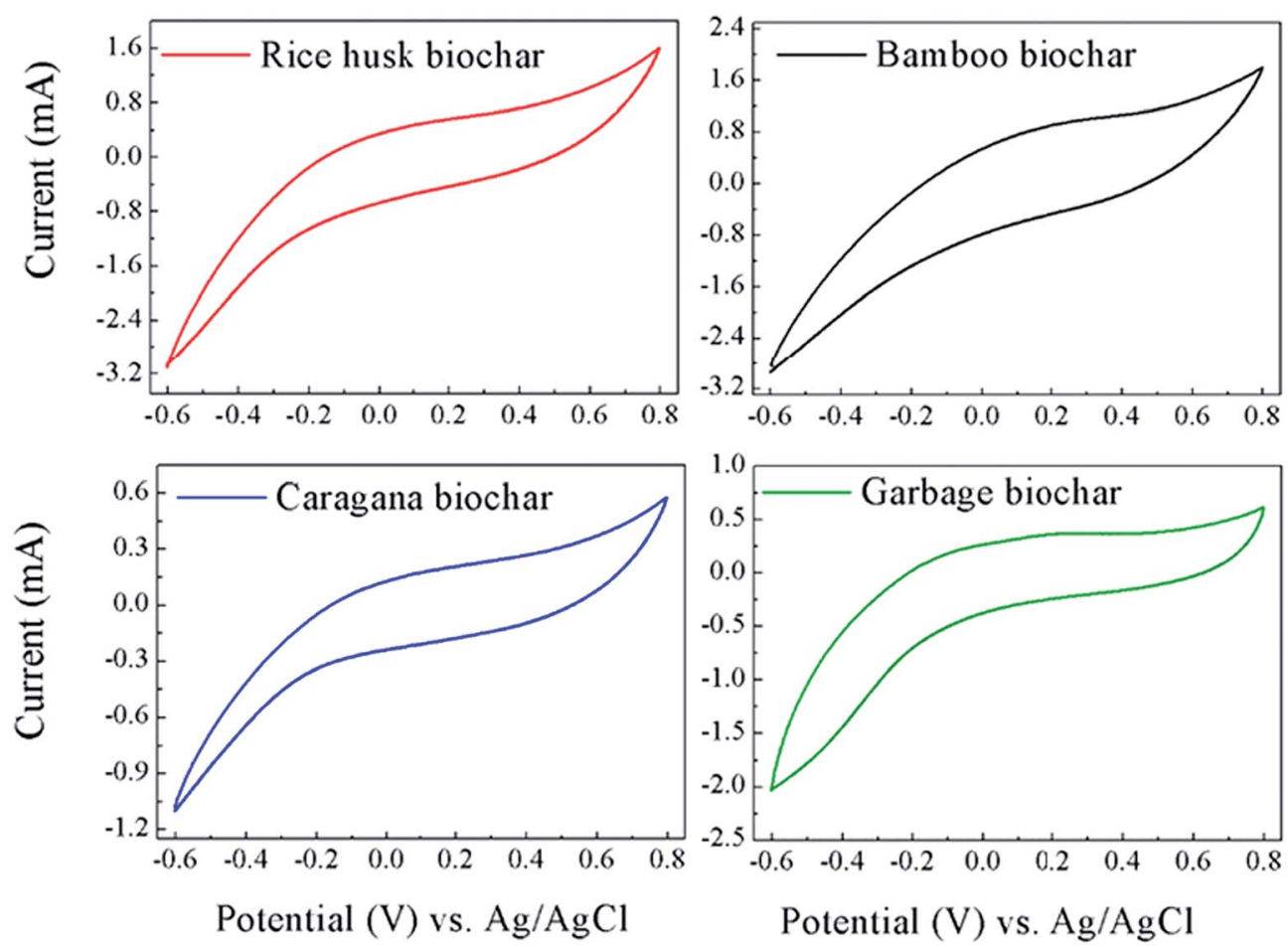

Fig. 5 Cyclic voltammograms (CVs) of the four biochars adhering on the working electrode in anaerobic medium. 
electrical conductor to accelerate the biodegradation of tetrachloroethene ${ }^{32}$ and active carbon could stimulate the direct electron transfer between microorganisms due to conductive properties. $^{33}$ Considering the ubiquity of black carbon and conductive minerals in the environment, it is likely that these processes occur naturally at many contamination sites.

There are several possible explanations for the promotion effect of biochar on electron transfer and microbial redox reactions. Firstly, both related bacteria and terminal electron acceptors (TEAs) could come into contact with biochar particles in biochar cultures. The presence of biochar might facilitate the electron transfer between the related bacteria and TEAs through the $\pi-\pi$ network of conductive graphite regions of biochar. ${ }^{\mathbf{1 6 , 1 9}}$ This could lead to the enhanced reduction of TEAs via accepting electrons. $^{34}$ Secondly, the redox-active moieties (such as quinone moieties and/or conjugated $\pi$-electron systems associated with condensed aromatic structures) harbored on biochar can reversibly accept and donate electrons, ${ }^{18,31,35}$ and might effectively mediate electron transfer and accelerate microbial reductive reaction of TEAs. Kappler et al. ${ }^{36}$ reported that biochar, due to its redox activity, could function as an electron shuttle to facilitate the electron transfer from Shewanella oneidensis MR-1 to $\mathrm{Fe}(\mathrm{III})$ minerals. Chen et al. ${ }^{35}$ further demonstrated that redox active biochar can serve as an electron shuttle to enhance anaerobic reduction debromination of $2,2^{\prime}, 4,4^{\prime}$-tetrabromodiphenyl ether (BDE-47) in mangrove sediment.

To better assess the redox-active moieties in the biochars, CV experiments were conducted by adhering the biochar on the graphite electrode to increase the redox reactivity between the biochars and electrode. Fig. 5 shows the CVs of the four biochars adhered on the working electrode, however no obvious redox peaks appeared in all of the biochars. Therefore, the CVs data also indicated that the redox-active moieties did not effectively exist in biochars and the enhancement of PCP dechlorination by biochar was probably not attributable to them.

In this biochar-dependent PCP-to-phenol dechlorinating culture, ETC or redox-active moieties in biochar do not seem to be as important as EC for microbial PCP dechlorination, although several studies have demonstrated the crucial role of redox-active moieties harbored in biochars for the microbial redox reactions. Further research is warranted to clearly elucidate how the EC of biochar affects the microbial redox metabolisms and clarify the dechlorination mechanisms of PCP in this dechlorinating culture depending on the caragana biochar.

\section{Conclusions}

Four biochars made from different materials were applied to promote the microbial reductive dechlorination of PCP, and their properties were characterized. The results indicated that only caragana biochar exhibited positive promotion activity. Notably, caragana biochar had the highest EC, while the EC values of the other biochars were three orders of magnitude lower. Elemental composition, Fourier transform infrared spectra, and CV analyses revealed that all biochars possessed similar structures and functional group classes. Furthermore, the results showed that biochars derived from different raw materials harbor different ECs, which might in turn influence their ability for microbial transformation of environmental pollutants. Further research should be carried out to clarify the mechanism of electron transfer between conductive biochars and microbes. These findings could help us to understand the impact of raw materials used for biochar on the bioremediation of environmental contaminants and utilize biochars in the future.

\section{Conflicts of interest}

There are no conflicts of interest to declare.

\section{Acknowledgements}

This work was supported by the Natural Science Foundation of China (No. 31400096, 41701346); the Fundamental Research Funds for the Central Universities (No. 2017QNA4045); and Open Research Fund of State Key Laboratory of Environmental Chemistry and Ecotoxicology, Research Center for EcoEnvironmental Sciences (No. KF2017-20).

\section{Notes and references}

1 M. Peper, M. Ertl and I. Gerhard, Am. J. Ind. Med., 1999, 35, 632-641.

2 C. Zhang and A. Katayama, Environ. Sci. Technol., 2012, 46, 6575-6583.

3 C. Zhang, D. Suzuki, Z. Li, L. Ye and A. Katayama, J. Biosci. Bioeng., 2012, 114, 512-517.

4 D. Zhang, C. Zhang, Z. Li, D. Suzuki, D. D. Komatsu, U. Tsunogai and A. Katayama, Bioresour. Technol., 2014, 164, 232-240.

5 S. Yang, A. Shibata, N. Yoshida and A. Katayama, Biotechnol. Bioeng., 2009, 102, 81-90.

6 C. Zhang, Z. Li, D. Suzuki, L. Ye, N. Yoshida and A. Katayama, Chemosphere, 2013, 92, 1343-1348.

7 D. Zhang, N. Zhang, X. Yu, Z. Zhang, S. Yang and C. Zhang, RSC Adv., 2017, 7, 6849-6855.

8 L. Beesley, E. Moreno-Jiménez, J. L. Gomez-Eyles, E. Harris, B. Robinson and T. Sizmur, Environ. Pollut., 2011, 159, 3269-3282.

9 D. Mohan, K. Abhishek, A. Sarswat, M. Patel, P. Singh and C. U. Pittman, RSC Adv., 2018, 8, 508-520.

10 M. Wang, Y. Zhu, L. Cheng, B. Andserson, X. Zhao, D. Wang and A. Ding, J. Environ. Sci., 2017, 63, 156-173.

11 X. Cao, L. Ma, Y. Liang, B. Gao and W. Harris, Environ. Sci. Technol., 2011, 45, 4884-4889.

12 M. Li, Z. Zhao, X. Wu, W. Zhou and L. Zhu, RSC Adv., 2017, 7, 2127-2136.

13 M. Uchimiya, L. H. Wartelle and V. M. Boddu, J. Agric. Food Chem., 2012, 60, 2989-2997.

$14 \mathrm{H}$. Wu and Q. Feng, J. Environ. Sci., 2017, 54, 346-357.

15 Y. Yan, M. Ma, X. Liu, W. Ma, M. Li and L. Yan, Int. Biodeterior. Biodegrad., 2017, 124, 119-127. 
16 S. Chen, A.-E. Rotaru, P. M. Shrestha, N. S. Malvankar, F. Liu, W. Fan, K. P. Nevin and D. R. Lovley, Sci. Rep., 2014, 4, 5019.

17 S. Y. Oh, J. G. Son and P. C. Chiu, Environ. Toxicol. Chem., 2013, 32, 501-508.

18 L. Klüpfel, M. Keiluweit, M. Kleber and M. Sander, Environ. Sci. Technol., 2014, 48, 5601-5611.

19 L. Yu, Y. Yuan, J. Tang, Y. Wang and S. Zhou, Sci. Rep., 2015, 5, 16221.

20 C. Zhang, D. Zhang, Z. Xiao, Z. Li, D. Suzuki and A. Katayama, Chemosphere, 2015, 131, 110-116.

21 P. Guo, C. Zhang, Y. Wang, X. Yu, Z. Zhang and D. Zhang, Environ. Pollut., 2018, 234, 107-114.

22 F. Widdel, G. W. Kohring and F. Mayer, Arch. Microbiol., 1983, 134, 286-294.

23 Y. Yuan, Y. Tao, S. Zhou, T. Yuan, Q. Lu and J. He, Bioresour. Technol., 2012, 116, 428-434.

24 F. J. Cervantes, J. Gonzalez-Estrella, A. Márquez, L. H. Alvarez and S. Arriaga, Bioresour. Technol., 2011, 102, 2097-2100.

25 C. M. Martínez, L. B. Celis and F. J. Cervantes, Appl. Microbiol. Biotechnol., 2013, 97, 9897-9905.

26 C. Zhang, D. Zhang, Z. Li, T. Akatsuka, S. Yang, D. Suzuki and A. Katayama, Environ. Sci. Technol., 2014, 48, 6318-6325.
27 X. Lu, J. Hanna and W. Johnson, Appl. Geochem., 2000, 15, 1019-1033.

28 O. Francioso, C. Ciavatta, C. Gessa, V. Tugnoli and S. Sanchez-Cortes, Soil Sci. Soc. Am. J., 1998, 62, 181-187.

29 K. B. Cantrell, P. G. Hunt, M. Uchimiya, J. M. Novak and K. S. Ro, Bioresour. Technol., 2012, 107, 419-428.

30 M. Tatzber, M. Stemmer, H. Spiegel, C. Katzlberger, G. Haberhauer, A. Mentler and M. H. Gerzabek, J. Plant Nutr. Soil Sci., 2007, 170, 522-529.

31 W. Xu, J. J. Pignatello and W. A. Mitch, Environ. Sci. Technol., 2013, 47, 7129-7136.

32 F. Aulenta, S. Rossetti, S. Amalfitano, M. Majone and V. Tandoi, ChemSusChem, 2013, 6, 433-436.

33 F. Liu, A.-E. Rotaru, P. M. Shrestha, N. S. Malvankar, K. P. Nevin and D. R. Lovley, Energy Environ. Sci., 2012, 5, 8982-8989.

34 J. He, K. R. Robrock and L. Alvarez-Cohen, Environ. Sci. Technol., 2006, 40, 4429-4434.

35 J. Chen, C. Wang, Y. Pan, S. S. Farzana and N. F. Tam, J. Hazard. Mater., 2018, 341, 177-186.

36 A. Kappler, M. L. Wuestner, A. Ruecker, J. Harter, M. Halama and S. Behrens, Environ. Sci. Technol. Lett., 2014, 1, 339-344. 